\title{
Minimum Action Solutions of Some Vector Field Equations
}

\author{
Haim Brezis ${ }^{1}$ and Elliott H. Lieb ${ }^{2, \star}$ \\ 1 Département de Mathématiques, Université Paris VI, 4, Place Jussieu, F-75230 Paris, \\ Cédex 05, France \\ 2 Departments of Mathematics and Physics, Princeton University, P.O. Box 708, Princeton, \\ NJ 08544, USA
}

\begin{abstract}
The system of equations studied in this paper is $-\Delta u_{i}=g^{i}(u)$ on $\mathbb{R}^{d}$, $d \geqq 2$, with $u: \mathbb{R}^{d} \rightarrow \mathbb{R}^{n}$ and $g^{i}(u)=\partial G / \partial u_{i}$. Associated with this system is the action, $S(u)=\int\left\{\frac{1}{2}|\nabla u|^{2}-G(u)\right\}$. Under appropriate conditions on $G$ (which differ for $d=2$ and $d \geqq 3$ ) it is proved that the system has a solution, $u \neq 0$, of finite action and that this solution also minimizes the action within the class $\{v$ is a solution, $v$ has finite action, $v \neq 0\}$.
\end{abstract}

\section{Introduction}

The purpose of this paper is to demonstrate the existence of solutions to a class of systems of partial differential equations that arises in several branches of mathematical physics (e.g. calculating lifetimes of metastable states, estimates of large order behavior of perturbation theory, Ginzburg-Landau theory, density of states in disordered systems). The systems to be considered are of the form

$$
-\Delta u_{i}(x)=g^{i}(u(x)), \quad i=1, \ldots, n .
$$

Furthermore, it will be shown that among the nonzero solutions to (1.1) there is one that minimizes the action, $S(u)$, associated with (1.1).

The meaning of the quantities in (1.1) is the following: $u \equiv\left(u_{1}, \ldots, u_{n}\right) \in \mathbb{R}^{n}$ and each $u_{i}: \mathbb{R}^{d} \rightarrow \mathbb{R}$ with $d \geqq 2$. We require that $u_{i}(x) \rightarrow 0$ as $|x| \rightarrow \infty$ in a weak sense described below (namely $u \in \mathscr{C}$ ). (Note: In some applications it is required that $u(x)$ $\rightarrow c=$ constant as $|x| \rightarrow \infty$ but, by redefining $u \rightarrow u-c$ and by redefining $g^{i}$, the problem can be reduced to the $u(x) \rightarrow 0$ case.) The $n$ functions $g^{i}: \mathbb{R}^{n} \rightarrow \mathbb{R}$ are the gradients of some function $G \in C^{1}\left(\mathbb{R}^{n} \backslash\{0\}\right)$, namely

$$
\begin{array}{ll}
g^{i}(u)=\partial G(u) / \partial u_{i}, & u \neq 0, \\
g^{i}(u)=0, & u=0,
\end{array}
$$

* Work partially supported by U.S. National Science Foundation Grant PHY-81-16101-A02 
and $G$ satisfies certain properties described in Sect. II $(d \geqq 3)$ and Sect. III $(d=2)$. In particular, we emphasize that $G(u)$ need not be differentiable at $u=0$ so that, for example, $G(u)$ could be $-|u|$ near $u=0$.

The Action associated with (1.1) is

$$
\begin{gathered}
S(u) \equiv K(u)-V(u), \\
K(u) \equiv \frac{1}{2} \int|\nabla u(x)|^{2} d x \equiv \frac{1}{2} \sum_{i} \int\left|\nabla u_{i}(x)\right|^{2} d x, \\
V(u) \equiv \int G(u(x)) d x .
\end{gathered}
$$

In general, $S(u)$ is not bounded below, and one of our goals is to show that, under suitable conditions, $S(u)>-\infty$ if $u$ satisfies (1.1) and that $S(u)$ actually has a minimum in the set of non-trivial solutions to (1.1). The word non-trivial (meaning $u \neq 0)$ is important; it will be shown later that when $d=2$ the function $u=0$ satisfies (1.1) and minimizes $S(u)$, but the non-trivial solutions to (1.1) all have $S(u)>0$. When $d \geqq 3$, the $u \equiv 0$ solution never has the minimum action.

The class of functions to which we shall restrict our investigation of (1.1) as an equation in $\mathscr{D}^{\prime}$ is

$$
\mathscr{C}=\left\{u \mid u \in L_{\mathrm{loc}}^{1}\left(\mathbb{R}^{d}\right), \nabla u \in L^{2}\left(\mathbb{R}^{d}\right), G(u) \in L^{1}\left(\mathbb{R}^{d}\right), \mu([|u|>\alpha])<\infty \text { for all } \alpha>0\right\} .(1
$$

Here, the symbol $[f>\alpha]$ denotes the set $\{x \mid f(x)>\alpha\}$. The same symbol, $[f>\alpha]$, will also be used to denote the characteristic function of this set. Lebesgue measure is denoted by $\mu$. The set

$$
\mathscr{E}=\left\{u \mid u \in \mathscr{C}, g(u) \in L_{\text {loc }}^{1}\left(\mathbb{R}^{d}\right), u \text { satisfies }(1.1) \text { in } \mathscr{D}^{\prime}, u \neq 0\right\}
$$

is the subset of $\mathscr{C}$ which we shall prove is non-empty and in which there is a $\bar{u}$ such that

$$
S(\bar{u}) \leqq S(u), \quad \text { all } \quad u \in \mathscr{E} .
$$

The solution of this problem was reported in 1983 and an outline of the proof was given [13]. The purpose of the present paper is to present all the details of the proof and certain additional refinements.

Probably the earliest general treatment of existence of finite action solutions to (1.1) was by Strauss [20] for $n=1, d \geqq 1$. (The case $n=1$ is called the scalar case.) While this work was very important because it introduced new techniques, it imposed severe restrictions on the function $G$. Moreover, Strauss did not explicitly consider the question of whether or not his solution to (1.1) minimized the action. Strauss and Vázquez extended this work to the vector case and to the "zero mass" case [22]. The next step was taken by Coleman et al. [10] who made an important contribution to the problem by their "constrained minimum method" which not only yields a solution for $d \geqq 3$ but also yields a minimum action solution. They discovered almost optimal assumptions on $G$ so that the problem has a solution, but their method for finding a minimum action solution was restricted in an essential way to $d \geqq 3$ and $n=1$. A detailed treatment of the Coleman, Glaser, Martin method, together with some improvements and other theorems useful in the analysis of this and related problems was given by Berestycki and Lions [5, 6]. Then, in the same generality, Berestycki and Lions [6] 
went on to prove the existence of infinitely many finite action solutions to (1.1). Strauss [20] also had results in this direction. (Infinitely many solutions for the socalled zero mass case was done in [7].) As before, all of this was for $n=1, d \geqq 3$.

In view of the aforementioned work, two natural extensions suggest themselves. One is to $d=2$ and the other is to $n>1$. We thank Ian Affleck for suggesting both problems to one of us (E.L.). Affleck was interested in the $d=2$ case for physical applications [1]. Some results for both $d=2$ and for $n \geqq 1$ were obtained by one of us (E.L.) in 1983, and these were subsequently strengthened in collaboration with H.B. to the level of generality given here and in [13]. Independently, in 1982, Berestycki, Gallouet and Kavian had solved the $d=2$, $n=1$ case (with stronger hypotheses than in the present paper; in particular they do not treat the zero mass case) and this was published recently [3] (see also [4]). [However, they also showed there are infinitely many solutions of (1.1) for $d=2$, $n=1$.]

The proofs for $n=1$ all relied on the fact that one could look for minima in the class of radial functions (by rearrangement inequalities), and that these functions have certain compactness properties [20,6]. For $n>1$, one can still restrict attention to radial solutions, although it is not known whether the minimum action solution lies in this class (because rearrangement inequalities are not applicable). Berestycki and Lions [5] showed how to prove the existence of radial solutions that minimize the action among all radial solutions of (1.1).

The extension to $n>1$ requires a new compactness device. In this paper, the heart of the matter is contained in Lemma 2.2. It should be noted that Lions has developed a general compactness principle $[15,16]$ which allows him to deal with the cases $d \geqq 2, n \geqq 1$.

\section{The Case of Three or More Dimensions}

\section{A. The Minimization Problem}

Let $G: \mathbb{R}^{n} \rightarrow \mathbb{R}$ be continuous with $G(0)=0$. In this subsection we shall consider a minimization problem that leads to (1.1) if $G$ happens to be differentiable, but here we shall make no assumptions about the differentiability of $G$. Here, and henceforth, $C>0$ will denote an inessential, positive constant. $G$ satisfies the following four conditions (2.2)-(2.5). [Note $G(u)$, not $|G(u)|$ in (2.2), (2.3).]

$$
\limsup _{|u| \rightarrow \infty}|u|^{-p} G(u) \leqq 0,
$$

where, for $d \geqq 3, p$ always denotes

$$
\begin{gathered}
p=2^{*}=2 d /(d-2), \\
\limsup _{|u| \rightarrow 0}|u|^{-p} G(u) \leqq 0, \\
G\left(u_{0}\right)>0 \text { for some } u_{0} \in \mathbb{R}^{n},
\end{gathered}
$$

$\left.\begin{array}{l}\text { For all } \gamma>0 \text { there exists } C_{\gamma} \text { such that for all } u, w \in \mathbb{R}^{n} \\ |G(u+w)-G(u)| \leqq \gamma\left[|G(u)|+|u|^{p}\right]+C_{\gamma}\left[|G(w)|+|w|^{p}+1\right] .\end{array}\right\}$ 
Remark 1. Condition (2.5) looks awkward, but it holds in several cases such as (2.6) or (2.7) or (2.8):

$$
\begin{aligned}
& \lim _{|u| \rightarrow \infty}|u|^{-p}|G(u)|=0, \\
& \left.\begin{array}{ll}
G \in C^{1}\left(\mathbb{R}^{n} \backslash\{0\}\right) & \text { and } g=\nabla G \text { satisfies } \\
|g(u)| \leqq C+C|u|^{p-1}, & \text { all } u \neq 0 .
\end{array}\right\} \\
& G \in C^{1}\left(\mathbb{R}^{n} \backslash\{0\}\right) \text { and } g=\nabla G \text { satisfies } \\
& |g(u)| \leqq C+C|u|^{q-1}, \quad \text { all } u \neq 0 \text { and } \\
& |u|^{p}+|G(u)| \geqq \alpha|u|^{q}-C, \quad \text { all } u \in \mathbb{R}^{n} \\
& \text { for some } q \geqq p \text {, and } C, \alpha>0 \text {. }
\end{aligned}
$$

The main result of this section is the solution to the following minimization problem. We define

$$
T=\inf \left\{\frac{1}{2} \int|\nabla u|^{2} \mid u \in \mathscr{C}, \int G(u) \geqq 1\right\} .
$$

Theorem 2.1. Assume (2.2)-(2.5). Then there exists $v \in \mathscr{C}$ such that

$$
\frac{1}{2} \int|\nabla v|^{2}=T
$$

and

$$
\int G(v)=1 \text {. }
$$

Remark 2. Using (2.4) it is easy to see that there is some $u \in \mathscr{C}$ such that $\int G(u)=1$.

Remark 3. Let $u \in L_{\text {loc }}^{1}$ and $\nabla u \in L^{2}$, such that $u \rightarrow 0$ as $|x| \rightarrow \infty$ in the weak sense of (1.6), namely $\mu([|u|>\alpha])<\infty$, all $\alpha>0$. Then $u \in L^{p}$ and $\|u\|_{p} \leqq C\|\nabla u\|_{2}$. Thus, the class $\mathscr{C}$ in (1.6) can be characterized (for $d \geqq 3$ ) as

$$
\mathscr{C}=\left\{u \mid u \in L^{p}\left(\mathbb{R}^{d}\right), \nabla u \in L^{2}\left(\mathbb{R}^{d}\right), G(u) \in L^{1}\left(\mathbb{R}^{d}\right)\right\} .
$$

To prove this, let $\chi_{n}(x)=\chi(x / n)$, where $\chi \in C_{0}^{\infty}$ and $\chi \equiv 1$ near 0 . Let $\varepsilon>0$ be fixed. Assume, provisionally, that $u \in \mathscr{C}$ and also $u \in L^{\infty}$. By Sobolev's inequality,

$$
\begin{aligned}
\left\|\chi_{n}(|u|-\varepsilon)_{+}\right\|_{p} & \leqq C\left\|\nabla \chi_{n}(|u|-\varepsilon)_{+}\right\|_{2} \\
& \leqq C\left\{\|\nabla u\|_{2}+\left[\int_{A}\left|\nabla \chi_{n}\right|^{2}\right]^{1 / 2}\right\} \\
& \leqq C\|\nabla u\|_{2}+C_{\varepsilon} / n,
\end{aligned}
$$

where $A=[|u|>\varepsilon]$ and $C_{\varepsilon}$ is some constant depending on $\varepsilon$. We conclude (in this $L^{\infty}$ case) by letting $n \rightarrow \infty$ and then $\varepsilon \rightarrow 0$. If $u \in \mathscr{C}$ but $u \notin L^{\infty}$, we may truncate $u$, then use the foregoing, and then remove the truncation by Fatou's lemma.

In the following, $\left\{u^{j}\right\}$ denotes a minimizing sequence for (2.9).

Lemma 2.1. There exist $\varepsilon, \delta>0$ such that for all $j, \mu\left(\left[\left|u^{j}\right|>\varepsilon\right]\right) \geqq \delta$.

Proof. Since $\nabla u^{j}$ is bounded in $L^{2}$, Sobolev's inequality implies that

$$
\left\|u^{j}\right\|_{p}^{p} \leqq C .
$$

Let $\gamma=1 /(2 C)$. By (2.2), (2.3) there exists $1>\varepsilon>0$ such that

$$
G(v) \leqq \gamma|v|^{p} \quad \text { for } \quad|v| \leqq \varepsilon \quad \text { or } \quad|v| \geqq 1 / \varepsilon .
$$


Thus, we have that

$$
\begin{aligned}
& 1 \leqq \int G\left(u^{j}\right) \leqq \gamma \int\left|u^{j}\right|^{p}\left\{\left[\left|u^{j}\right| \leqq \varepsilon\right]+\left[\left|u^{j}\right| \geqq 1 / \varepsilon\right]\right\}+\int G\left(u^{j}\right)\left[\varepsilon<\left|u^{j}\right|<1 / \varepsilon\right] \\
& \quad \leqq \frac{1}{2}+C_{\varepsilon} \mu\left(\left[\left|u^{j}\right|>\varepsilon\right]\right) .
\end{aligned}
$$

This implies the lemma with $\delta=1 /\left(2 C_{\varepsilon}\right)$.

Next, we recall the following [14]:

Lemma 2.2. Let $v$ be a function such that $v \in L_{\mathrm{loc}}^{1}, \nabla v \in L^{2},\|\nabla v\|_{2} \leqq C$ and $\mu([|v|>\varepsilon]) \geqq \delta>0$. Then, there exists a shift $T_{y} v(x)=v(x+y)$ such that, for some constant $\alpha=\alpha(C, \delta, \varepsilon)>0, \mu\left(B \cap\left[\left|T_{y} v\right|>\varepsilon / 2\right]\right)>\alpha$, where $B=\left\{x \in \mathbb{R}^{d}|| x \mid \leqq 1\right\}$.

Using Lemmas 2.1 and 2.2 we can shift each $u^{j}$ in such a way that $\mu\left(B \cap\left[\left|T_{y_{j}} u^{j}\right|>\varepsilon / 2\right]\right) \geqq \alpha$, where $\alpha>0$ is independent of $j$. Thus, we may assume without loss of generality that $\mu\left(B \cap\left[\left|u^{j}\right|>\varepsilon / 2\right]\right) \geqq \alpha$. After extracting a subsequence we may also assume that (cf. (2.12))

$$
\begin{gathered}
u^{j} \rightarrow u \text { weakly in } L^{p}, \nabla u^{j} \rightarrow \nabla u \text { weakly in } L^{2}, \\
u^{j} \rightarrow u \text { a.e. on } \mathbb{R}^{d}, \mu(B \cap[|u| \geqq \varepsilon / 2]) \geqq \alpha .
\end{gathered}
$$

Finally, we have $G(u) \in L^{1}$. To prove this, let us write

$$
G=G_{+}-G_{-} \quad \text { with } G_{+}=\operatorname{Max}\{G, 0\} \quad \text { and } \quad G_{-}=\operatorname{Max}\{-G, 0\} .
$$

We have

$$
\begin{aligned}
\int G_{+}\left(u^{j}\right) \leqq & \gamma \int\left|u^{j}\right|^{p}\left\{\left[\left|u^{j}\right| \leqq \varepsilon\right]+\left[\left|u^{j}\right| \geqq 1 / \varepsilon\right]\right\} \\
& +\int G_{+}\left(u^{j}\right)\left[\varepsilon<\left|u^{j}\right|<1 / \varepsilon\right] \leqq \text { const } .
\end{aligned}
$$

(The last integral is uniformly bounded because $\left\|u^{j}\right\|_{p}<C \Rightarrow \mu\left(\left[\left|u^{j}\right|>\varepsilon\right]\right)$ $<(C / \varepsilon)^{p}$; moreover, $G_{+}(\cdot)$ is bounded on $(\varepsilon, 1 / \varepsilon)$ since $G_{+}$is continuous.) We also have $\int G_{-}\left(u^{j}\right) \leqq \int G_{+}\left(u^{j}\right)-1$. Hence, $\int\left|G\left(u^{j}\right)\right| \leqq$ const, and we deduce from Fatou's lemma that $G(u) \in L^{1}$. Thus, $u \in \mathscr{C}$.

We conclude the proof of Theorem 2.1 with

Lemma 2.3. The limit function satisfies $\int G(u)=1$ and $\frac{1}{2} \int|\nabla u|^{2}=T$, where $T$ is defined in (2.9).

Remark 4. It follows from Lemma 2.3 that in fact $\nabla u^{j} \rightarrow \nabla u$ strongly in $L^{2}$ and thus $u^{j} \rightarrow u$ strongly in $L^{p}$.

Proof of Lemma 2.3. It is easily seen by scaling [i.e. $v(x) \rightarrow v(\lambda x)$ ] that

$$
\frac{1}{2} \int|\nabla v|^{2} \geqq T\left[\int G(v)\right]^{(d-2) / d}, \quad \text { all } v \in \mathscr{C} \text { with } \int G(v)>0 .
$$

Let $\phi \in L^{p}$ with $G(\phi) \in L^{1}$ and with $\phi$ having compact support. We claim that, as $j \rightarrow \infty$

$$
\int G\left(u^{j}+\phi\right) \geqq 1+\int G(u+\phi)-\int G(u)+o(1) .
$$

[Note that the integrals in (2.15) make sense because of (2.5).] 
Verification of (2.15). Let $K=\operatorname{Supp} \phi$; we have

$$
\begin{aligned}
\int G\left(u^{j}+\phi\right) & =\int_{K} G\left(u^{j}+\phi\right)+\int_{\sim K} G\left(u^{j}\right) \geqq 1+\int_{K}\left[G\left(u^{j}+\phi\right)-G\left(u^{j}\right)\right] \\
& =1+\int_{K}[G(u+\phi)-G(u)]+o(1) .
\end{aligned}
$$

The last equality follows from Egorov's (or Vitali's) lemma. Indeed, given $\varepsilon>0$ we fix $\gamma>0$ small enough so that

$$
\gamma \int\left\{\left|G\left(u^{j}\right)\right|+\left|u^{j}\right|^{p}\right\}<\varepsilon / 2 .
$$

By (2.5) we have that

$$
\int_{A}\left|G\left(u^{j}+\phi\right)-G\left(u^{j}\right)\right| \leqq \varepsilon / 2+C_{\gamma} \int_{A}\left[|G(\phi)|+|\phi|^{p}+1\right] \leqq \varepsilon
$$

for any set $A \subset K$ with $\mu(A)<\delta$ ( $\delta$ small enough). Thus we have established (2.15).

Suppose now that $\phi \in H^{1}, \phi$ has compact support, $G(\phi) \in L^{1}$ and

$$
\int[G(u+\phi)-G(u)]>-1 \text {. }
$$

For $j$ large enough we may insert $v=u^{j}+\phi$ in (2.14) and, in the limit, we find that

$$
T+\int \nabla u \cdot \nabla \phi+\frac{1}{2} \int|\nabla \phi|^{2} \geqq T\left[1+\int G(u+\phi)-\int G(u)\right]^{1-2 / d} .
$$

That is,

$$
T+\frac{1}{2} \int|\nabla(u+\phi)|^{2}-\frac{1}{2} \int|\nabla u|^{2} \geqq T\left[1+\int G(u+\phi)-\int G(u)\right]^{1-2 / d} .
$$

Let $\lambda>0$ be fixed. We can find a mapping $S: \mathbb{R}^{d} \rightarrow \mathbb{R}^{d}$, bijective with $S$ and $S^{-1}$ smooth such that

$$
S(x)= \begin{cases}\lambda x, & |x|<1 \\ x, & |x|>R\end{cases}
$$

(for some $R$ depending on $\lambda$ ). Set $S_{n}(x)=n S(x / n)$ and $\phi_{n}(x)=u\left(S_{n}(x)\right)-u(x)$, so that $\phi_{n} \in H^{1}$ and $\phi_{n}$ has compact support and $G\left(\phi_{n}\right) \in L^{1}$. [The last assertion is obtained by choosing $u=\phi_{n}(x), w=u(x)$ in (2.5).] We claim that as $n \rightarrow \infty$

$$
\int G\left(u+\phi_{n}\right)=\int G(u(\lambda x)) d x+o(1)=\lambda^{-d} \int G(u)+o(1),
$$

and

$$
\int\left|\nabla\left(u+\phi_{n}\right)\right|^{2}=\int|\nabla[u(\lambda x)]|^{2} d x+o(1)=\lambda^{2-d} \int G(u)+o(1) .
$$

Indeed we write

$$
\int G\left(u+\phi_{n}\right)=\int G\left(u\left(S_{n}(x)\right)\right) d x=\int G(u(y)) J_{n}(y) d y,
$$

where $J_{n}$ denotes the Jacobian determinant of the mapping $y \rightarrow S_{n}^{-1}(y)$; it is easy to see that $\left|J_{n}\right| \leqq C, C$ independent of $n$, and $J_{n}(y) \rightarrow \lambda^{-d}$ as $n \rightarrow \infty$ for all $y$. Thus (2.18) follows by dominated convergence. The same argument applies to (2.19). We fix $\lambda>0$ with $|\lambda-1|$ so small that $\left(\lambda^{-d}-1\right) \int G(u)>-1$. Thus $\phi=\phi_{n}$ satisfies $(2.16)$ for $n$ large enough. Hence (2.17) holds for $\phi=\phi_{n}$ and in the limit (as $n \rightarrow \infty$ ) we obtain

$$
T+\frac{1}{2}\left(\lambda^{2-d}-1\right) \int|\nabla u|^{2} \geqq T\left[1+\left(\lambda^{-d}-1\right) \int G(u)\right]^{1-2 / d} .
$$


Finally we choose $\lambda=1 \pm \varepsilon$ in (2.20) and, as $\varepsilon \rightarrow 0$, we see that $\frac{1}{2} \int|\nabla u|^{2}=T \int G(u)$. Since $u \neq 0$ we have $\int G(u)>0$, and we deduce from (2.14) (applied to $v=u$ ) that $\int G(u) \geqq 1$. On the other hand, since $\nabla u^{j} \rightarrow \nabla u$ weakly in $L^{2}$, we obtain, by lower semicontinuity, that $\frac{1}{2} \int|\nabla u|^{2} \leqq T$. Therefore $\int G(u)=1$ and $\frac{1}{2} \int|\nabla u|^{2}=T$. This concludes the proof of Theorem 2.1.

\section{B. Further Properties of $u$}

Throughout this section we assume that $G$ is differentiable on $\mathbb{R}^{n} \backslash\{0\}$. More precisely, let $G: \mathbb{R}^{n} \rightarrow \mathbb{R}$ be continuous (on all of $\mathbb{R}^{n}$ ) with $G(0)=0$. Assume that $G$ satisfies (2.2)-(2.4) and $G \in C^{1}\left(\mathbb{R}^{n} \backslash\{0\}\right)$. We set

$$
g(v)=\left\{\begin{array}{lll}
\nabla G(v) & \text { if } & v \neq 0 \\
0 & \text { if } & v=0
\end{array}\right.
$$

We assume (2.8). For every $v \in \mathscr{C}$ we define its action to be $S(v)=\frac{1}{2} \int|\nabla v|^{2}-\int G(v)$.

Theorem 2.2. Let $u$ be given by Theorem 2.1. Then after some appropriate scaling,

Moreover,

$$
\begin{gathered}
\underline{u}(x)=u(\theta x),(\theta>0), \underline{u} \text { satisfies } \\
-\Delta \underline{u}=g(\underline{u}) \text { in } \mathscr{D}^{\prime} .
\end{gathered}
$$

$$
0<S(\underline{u}) \leqq S(v) \text {, all } v \in \mathscr{C} \cap L_{\mathrm{loc}}^{\infty}, v \neq 0,-\Delta v=g(v) \text { in } \mathscr{D}^{\prime} .
$$

[In some cases, any solution $v$ is automatically in $L_{\mathrm{loc}}^{\infty}$ (see Theorem 2.3).]

Proof. Fix $\phi \in C_{0}^{\infty}$. We see easily by dominated convergence that as $t \rightarrow 0$

$$
\int[G(u+t \phi)-G(u)][u \neq 0]=t \int[g(u) \cdot \phi][u \neq 0]+o(t) .
$$

Here we use (2.8). Also, we have that

$$
\int|G(u+t \phi)-G(u)|[u=0] \leqq C t \int|\phi|[u=0]+o(t) .
$$

From (2.14), and using (2.22) and (2.23) we deduce that, for $|t|$ small enough,

$$
\begin{aligned}
\frac{1}{2} \int|\nabla(u+t \phi)|^{2} & \geqq T\left\{\int G(u+t \phi)\right\}^{1-2 / d} \geqq T\left\{1+t \int g(u) \cdot \phi-C t \int|\phi|[u=0]+o(t)\right\}^{1-2 / d} \\
& \geqq T\left\{1+t\left(\frac{d-2}{d}\right) \int g(u) \cdot \phi-C t\left(\frac{d-2}{d}\right) \int|\phi|[u=0]+o(t)\right\} .
\end{aligned}
$$

Consequently,

$$
\left|\int \nabla u \cdot \nabla \phi-T\left(\frac{d-2}{d}\right) \int g(u) \cdot \phi\right| \leqq C \int|\phi|[u=0], \quad \text { all } \phi \in C_{0}^{\infty} .
$$

We deduce from the Riesz representation theorem that there exists some $h \in L^{\infty}$ such that

$$
-\Delta u=T\left(\frac{d-2}{d}\right) g(u)+h[u=0] \text { in } \mathscr{D}^{\prime} .
$$

Finally, we have $u \in L_{\mathrm{loc}}^{q}($ by $(2.8))$ and $g(u) \in L_{\text {loc }}^{q /(q-1)}$. We deduce from the elliptic regularity theory that $u \in W_{\mathrm{loc}}^{2, q /(q-1)}[$ since $q /(q-1)>1]$. Therefore $\Delta u=0$ a.e. on 
the set $[u=0]$ (see [19] or [11]). Hence we have proved that

$$
-\Delta u=T\left(\frac{d-2}{d}\right) g(u) \text { in } \mathscr{D}^{\prime}
$$

and therefore that $\underline{u}(x)=u(\theta x)$ satisfies $(2.21)$ with $\theta^{2}=d[(d-2) T]^{-1}$. In order to complete the proof of Theorem 2.1 we must establish Pohozaev's identity [18] in a setting slightly more general than usual.

Lemma 2.4. Assume $G \in C^{1}\left(\mathbb{R}^{n} \backslash\{0\}\right)$ and let $v \in \mathscr{C} \cap L_{\text {loc }}^{\infty}$ be any solution of $(1.1)$ in $\mathscr{D}^{\prime}$. Then

$$
\int|\nabla v|^{2}=\frac{2 d}{d-2} \int G(v)
$$

Proof. Since $v \in L_{\mathrm{loc}}^{\infty}$, it follows from (1.1) and the elliptic regularity theory that $v \in W_{\text {loc }}^{2, t}$, all $t<\infty$. Note that $\partial G(v) / \partial x_{i}=g(v) \cdot \partial v / \partial x_{i}$ in $\mathscr{D}^{\prime}$. Indeed, choose a smoothing sequence $G_{k}$ for $G$ so that $G_{k} \rightarrow G$ uniformly on compact sets of $\mathbb{R}^{n}$ and $g_{k}=\nabla G_{k}$ tends to $g$ pointwise on $\mathbb{R}^{n} \backslash\{0\}$. We have $\partial G_{k}(v) / \partial x_{i}=g_{k}(v) \cdot \partial v / \partial x_{i}$, and thus, for $\phi \in C_{0}^{\infty}$,

$$
\int \frac{\partial}{\partial x_{i}}\left(G_{k}(v)\right) \phi=-\int G_{k}(v) \frac{\partial \phi}{\partial x_{i}} \rightarrow \int G(v) \frac{\partial \phi}{\partial x_{i}}
$$

and

$$
\int g_{k}(v) \cdot \frac{\partial v}{\partial x_{i}} \phi \rightarrow \int g(v) \cdot \frac{\partial v}{\partial x_{i}} \phi
$$

by dominated convergence (recall that $\partial v / \partial x_{i}=0$ a.e. on the set $[v=0]$ ). Next we multiply Eq. (1.1) by $\phi \sum_{i} x_{i} \partial v / \partial x_{i}$, where $\phi \in C_{0}^{\infty}$. Note that

$$
\int \phi g(v) \cdot \sum_{i} x_{i} \frac{\partial v}{\partial x_{i}}=-d \int G(v) \phi-\int G(v) \sum_{i} x_{i} \frac{\partial \phi}{\partial x_{i}},
$$

while

$$
-\int \phi \Delta v \cdot \sum_{i} x_{i} \frac{\partial v}{\partial x_{i}}=\left(1-\frac{d}{2}\right) \int\left\{\phi|\nabla v|^{2}+\sum_{i, j} \frac{\partial \phi}{\partial x_{j}} x_{i} \frac{\partial v}{\partial x_{i}} \cdot \frac{\partial v}{\partial x_{j}}\right\}-\frac{1}{2} \int \sum_{i} \frac{\partial \phi}{\partial x_{i}} x_{i}|\nabla v|^{2}
$$

Finally we choose $\phi(x)=\phi_{n}(x)=\phi_{1}(x / n)$, where $\phi_{1}$ is any function in $C_{0}^{\infty}$ such that $\phi_{1}(x)=1$ for $|x|<1$ and $\phi_{1}(x)=0$ for $|x|>2$. As $n \rightarrow \infty$ we obtain (2.24).

Proof of Theorem 2.2 Concluded. We have

$$
\int|\nabla \underline{u}|^{2}=\frac{2 d}{(d-2)} \int G(\underline{u}), \quad \int|\nabla v|^{2}=\frac{2 d}{(d-2)} \int G(v),
$$

and on the other hand we also have

$$
\frac{1}{2} \int|\nabla \underline{u}|^{2}=T\left[\int G(\underline{u})\right]^{1-2 / d}, \quad \frac{1}{2} \int|\nabla v|^{2} \geqq T\left[\int G(v)\right]^{1-2 / d} .
$$


Combining these relations we see that $\int G(v) \geqq \int G(\underline{u})$. However,

$$
\begin{aligned}
& S(v)=\frac{1}{2} \int|\nabla v|^{2}-\int G(v)=\frac{2}{(d-2)} \int G(v), \\
& S(\underline{u})=\frac{1}{2} \int|\nabla \underline{u}|^{2}-\int G(\underline{u})=\frac{2}{(d-2)} \int G(\underline{u}),
\end{aligned}
$$

and we obtain $0<S(\underline{u}) \leqq S(v)$ for all $v \in \mathscr{C} \cap L_{\mathrm{loc}}^{\infty}, v \neq 0,-\Delta v=g(v)$ in $\mathscr{D}^{\prime}$.

\section{Regularity and Behavior at Infinity}

In this section we shall only assume that $g: \mathbb{R}^{n} \rightarrow \mathbb{R}^{n}$ is any mapping bounded on bounded sets and such that $g(v) \cdot v \leqq C|v|+C|v|^{p}$, all $v \in \mathbb{R}^{n}$.

Theorem 2.3. Let $v \in \mathscr{C}$ with $g(v) \in L_{\mathrm{loc}}^{1}$ be any solution of $-\Delta v=g(v)$ in $\mathscr{D}^{\prime}$. Then

$$
v \in W_{\mathrm{loc}}^{2, q} \text {, all } q<\infty \text { (and consequently } v \in C_{\mathrm{loc}}^{1, \alpha} \text { for all } \alpha<1 \text { ) }
$$

and

$$
v \in L^{\infty} \quad \text { with } \quad \lim _{|x| \rightarrow \infty} v(x)=0 .
$$

Assume, in addition, that

$$
g(v) \cdot v \leqq-C|v|^{r}, \quad \text { all } v \in \mathbb{R}^{n} \text { with }|v|<\delta,
$$

for some constants $C>0, \delta>0,1 \leqq r \leqq 2$. Then

$$
\begin{gathered}
\text { if } r=2, v(x) \text { decays exponentially as }|x| \rightarrow \infty, \\
\text { if } 1 \leqq r<2, v(x) \text { has compact support. }
\end{gathered}
$$

Proof. By Kato's inequality (see Kato [12]) we have

$$
\Delta|v| \geqq \Delta v \cdot \frac{v}{|v|} \text { in } \mathscr{D}^{\prime},
$$

and thus

$$
-\Delta|v| \leqq-\Delta v \cdot \frac{v}{|v|}=\frac{g(v) \cdot v}{|v|} \leqq C+C|v|^{p-1} .
$$

Therefore

$$
-\Delta|v|+|v| \leqq C+C|v|^{p-1} \leqq C+A|v|
$$

where

$$
A=C|v|^{p-2}[|v| \geqq 1],
$$

so that $A \in L^{d / 2}$ and $\mu(\operatorname{Supp} A)<\infty$. We deduce from (2.30) that

$$
|v| \leqq Y *(C+A|v|) \leqq C+[Y *(A|v|)],
$$

where $Y$ denotes the Yukawa potential (recall that $Y \in L^{1}$ ). 
Applying Lemma A.1 (in the appendix) with $\alpha=d /(d-2)$ and $\beta=2 d /(d-2)$, we see that

$$
\int_{B}|v|^{q}<\infty, \quad \text { all } B \text { with } \mu(B)<\infty, \text { all } q<\infty .
$$

In order to prove (2.26) we note that

$$
-\Delta|v|^{2}=-2 v \cdot \Delta v-2|\nabla v|^{2} \leqq 2 v \cdot g(v)
$$

Given $\varepsilon>0$, we have, for some $\delta>0$,

$$
-\Delta|v|^{2}+|v|^{2} \leqq C|v|+C|v|^{p} \leqq \varepsilon+C|v|^{p}[|v|>\delta]
$$

and thus

$$
|v|^{2} \leqq C \varepsilon+(Y * \phi)
$$

where $\phi=C|v|^{p}[|v|>\delta]$.

From (2.31) we deduce that $\phi \in L^{q}$, all $q<\infty$. Since, on the other hand, $Y \in L^{t}$ for all $1 \leqq t<d /(d-2)$, it follows that $(Y * \phi)(x) \rightarrow 0$ as $|x| \rightarrow \infty$. Using (2.33) we obtain $v \in L^{\infty}$ and

$$
\limsup _{|x| \rightarrow \infty}|v(x)|^{2} \leqq C \varepsilon .
$$

This implies (2.26) since $\varepsilon$ is arbitrary.

Therefore we have $g(v) \in L^{\infty}$ and consequently $v \in W_{\text {loc }}^{2, q}$ for all $q<\infty$.

Finally we assume (2.27). Combining (2.26), (2.27), and (2.32) we see that

$$
-\Delta|v|^{2}+2 C|v|^{r} \leqq 0 \text { for }|x|>R \text {, }
$$

( $R$ large enough). We easily deduce (2.28) and (2.29) from (2.34) by comparison with radial supersolutions. (When $r=2$ this is standard, when $1 \leqq r<2$, see e.g. BenilanBrezis-Crandall [2]. A systematic survey of available methods for proving compact support can be found in the book of Diaz [23].)

\section{The Two-Dimensional Case}

Let $G: \mathbb{R}^{n} \rightarrow \mathbb{R}$ be continuous with $G(0)=0$, and $G \in C^{1}\left(\mathbb{R}^{n} \backslash\{0\}\right)$. We set

$$
g(v)=\left\{\begin{array}{lll}
\nabla G(v) & \text { if } & v \neq 0 \\
0 & \text { if } & v=0
\end{array}\right.
$$

We make the following assumptions

$$
\begin{gathered}
G(v)<0 \text { for } 0<|v| \leqq \varepsilon \text { for some } \varepsilon>0, \\
G\left(v_{0}\right)>0 \text { for some } v_{0}, \\
|g(v)| \leqq C+C|v|^{p-1}, \text { for all } v \text {, for some } 1<p<\infty .
\end{gathered}
$$

The class $\mathscr{C}$ of functions is given in (1.6).

Theorem 3.1. Assume (3.1), (3.2), (3.3). Then

$$
T=\operatorname{Inf}\left\{\frac{1}{2} \int|\nabla v|^{2} \mid v \in \mathscr{C}, v \neq 0, \int G(v) \geqq 0\right\}
$$


is achieved by some $u \in \mathscr{C}, u \neq 0$ such that $\int G(u)=0$. Moreover $u$ satisfies

$$
-\Delta u=g(u) \text { in } \mathscr{D}^{\prime} \text {, }
$$

and

$$
0<S(u) \leqq S(v),
$$

for all $v \in \mathscr{C}$ such that $v \neq 0$ and $-\Delta v=g(v)$ in $\mathscr{D}^{\prime}$.

It is important to note that Theorem 3.1 states that the unique minimizer of $S(u)$ in the set of functions that are in $\mathscr{C}$ and that satisfy (1.1) is, in fact, $u \equiv 0$. The existence of this trivial solution of (1.1) of lowest action is special to $d=2$. It is the chief difficulty in the two-dimensional case for the obvious reason that the minimum of $\int|\nabla u|^{2}$ with $\int G(u)=0$ would be $u \equiv 0$. Therefore we must impose the extra condition $u \neq 0$. (Independently, Keller [21] introduced the $u \neq 0$ constraint, but for $d \geqq 3$. Berestycki et al. $[3,4]$ used it for $d=2$.)

We do not have a general result (as in the $d \geqq 3$ case) for the existence of a minimum in (3.4) without assuming the differentiability of $G$ on $\mathbb{R}^{n} \backslash\{0\}$. However, if we assume that for some $\varepsilon>0$,

$$
\sup _{0 \leqq t \leqq 1}|G(t v)| \leqq C|G(v)|
$$

for all $|v|<\varepsilon$, then we can prove the existence of a minimizing $u$ for (3.4) under the assumptions (3.1), (3.2) and $|v|^{-p} G(v) \rightarrow 0$, as $|v| \rightarrow \infty$ for some fixed $p<\infty$.

Proof. Let $\left\{u^{j}\right\}$ be a minimizing sequence for (3.4). Note that $\mu\left(\left[\left|u^{j}\right|>\varepsilon\right]\right)>0$, since $u^{j} \neq 0$ and $\int G\left(u^{j}\right) \geqq 0$. On the other hand the expression $\int|\nabla u|^{2}$ is invariant under scaling. Thus we may always assume that

$$
\mu\left(\left[\left|u^{j}\right|>\varepsilon\right]\right)=1 .
$$

Also, after a shift, we may assume that

$$
\mu\left(B \cap\left[\left|u^{j}\right|>\varepsilon / 2\right]\right) \geqq \alpha>0,
$$

where $B$ is the unit ball (the argument is the same as for $d \geqq 3$ ).

The following lemma is needed in the proof of Theorem 3.1.

Lemma 3.1. We have that

$$
\int\left|u^{j}\right|^{q}\left[\left|u^{j}\right|>\varepsilon\right] \leqq C_{q}, \quad \text { all } q<\infty, \text { all } j .
$$

Proof. First we claim that

$$
\begin{gathered}
\|\phi\|_{q} \leqq C_{q}\|\nabla \phi\|_{2} \mu(\operatorname{Supp} \phi)^{1 / q}, \text { for all } 1 \leqq q<\infty, \\
\text { all } \phi \in L_{\mathrm{loc}}^{1}, \nabla \phi \in L^{2}, \mu(\operatorname{Supp} \phi)<\infty .
\end{gathered}
$$

The conclusion of Lemma 3.1 follows by choosing $\phi=\left(\left|u^{j}\right|-\varepsilon\right)_{+}$in (3.10), and we obtain $\left\|\left(\left|u^{j}\right|-\varepsilon\right)_{+}\right\|_{q} \leqq C_{q}\left\|\nabla u^{j}\right\|_{2}$, which implies (3.9). For the proof of (3.10) we argue as follows:

Step (i). We start with the well-known inequality

$$
\|\phi\|_{2} \leqq C\|\nabla \phi\|_{1}, \quad \text { all } \phi \in C_{0}^{1} .
$$

(See e.g. Nirenberg [17].) 
Step (ii). Inserting $\phi^{2}, \phi^{3}, \ldots, \phi^{n}, \ldots$ in (3.11) and interpolating, we find

$$
\|\phi\|_{q} \leqq C_{q}\|\nabla \phi\|_{1}^{\alpha}\|\nabla \phi\|_{2}^{1-\alpha},
$$

with $\alpha=2 / q, q<\infty$, all $\phi \in C_{0}^{1}$.

Step (iii). Smoothing by convolution, we see that (3.12) holds when $\phi \in L^{\infty}, \nabla \phi \in L^{2}$ and $\phi$ has compact support.

Step (iv). Inequality (3.12) still holds when $\phi \in L^{\infty}, \nabla \phi \in L^{2}$ and $\mu(\operatorname{Supp} \phi)<\infty$. Indeed use step (iii) with $\left(\chi_{n} \phi\right)$, where $\chi_{n}(x)=\chi_{1}(x / n)$ and $\chi_{1} \in C^{1}$ with $\chi_{1}(x)=1$ for $|x|<1$. Note that $\left\|\phi \nabla \chi_{n}\right\|_{1} \rightarrow 0$ and $\left\|\phi \nabla \chi_{n}\right\|_{2} \rightarrow 0$.

Step (v). Inequality (3.12) is valid for $\phi \in L_{\mathrm{loc}}^{1}, \nabla \phi \in L^{2}$ and $\mu(\operatorname{Supp} \phi)<\infty$. Indeed, we can use Step (iv) on truncated $\phi$ 's.

Step (vi). We obtain (3.10) from (3.12) by the Cauchy-Schwarz inequality.

Returning now to Theorem 3.1, we deduce from Lemma 3.1 that $\left\|u^{j}\right\|_{L^{q}(Q)} \leqq C(q, Q)$, all $q<\infty$, all $Q$ bounded. We may now extract a subsequence (still denoted by $\left\{u^{j}\right\}$ ) such that

$$
\begin{gathered}
u^{j} \rightarrow u \text { in } L^{q}(Q), \text { all } q<\infty, \text { all } Q \text { bounded, } \\
u^{j} \rightarrow u \text { a.e. on } \mathbb{R}^{2}, \\
\nabla u^{j} \rightarrow \nabla u \text { weakly in } L^{2}\left(\mathbb{R}^{2}\right), \\
\mu(B \cap[|u| \geqq \varepsilon / 2]) \geqq \alpha>0 \text { (in particular } u \neq 0), \\
\mu([|u| \geqq \varepsilon]) \leqq 1 .
\end{gathered}
$$

Moreover, we have $G(u) \in L^{1}$. Indeed, writing $G=G_{+}-G_{-}$, we have that

$$
\int G_{+}\left(u^{j}\right)=\int G_{+}\left(u^{j}\right)\left[\left|u^{j}\right|>\varepsilon\right] \leqq \int C\left|u^{j}\right|^{p}\left[\left|u^{j}\right|>\varepsilon\right] \leqq C
$$

[here we use (3.3)]. On the other hand $\int G_{-}\left(u^{j}\right) \leqq \int G_{+}\left(u^{j}\right)$, and therefore $\int\left|G\left(u^{j}\right)\right| \leqq C$, which implies, by Fatou, that $G(u) \in L^{1}$.

We also deduce that $u \in \mathscr{C}$ since $\mu([|u|>\varepsilon]) \leqq 1$ and $G(u) \in L^{1}$ [here we use assumption (3.1)].

Note that for any set $B$ of finite measure we have

$$
\int_{B}\left|u^{j}\right|^{q} \leqq C(q,|B|), \quad \text { all } q<\infty,
$$

and, also in the limit

$$
\int_{B}|u|^{q} \leqq C(q,|B|), \quad \text { all } q<\infty .
$$

Indeed we may write

$$
\int_{B}\left|u^{j}\right|^{q} \leqq \int\left|u^{j}\right|^{q}\left[\left|u^{j}\right| \geqq \varepsilon\right]+\int_{B} \varepsilon^{q} \leqq C+\varepsilon^{q} \mu(B) .
$$

Let us introduce the class of functions from $\mathbb{R}^{2} \rightarrow \mathbb{R}^{n}$,

$$
\mathscr{K}=\left\{\phi \mid \phi \in L_{\mathrm{loc}}^{1}, \nabla \phi \in L^{2}, \mu(\operatorname{Supp} \phi)<\infty\right\} .
$$

We recall that by (3.10) $\phi \in \mathscr{K}$ implies $\phi \in L^{q}\left(\mathbb{R}^{2}\right)$, all $q, 1 \leqq q<\infty$. 
Lemma 3.2. Let $\phi \in \mathscr{K}$ be such that

$$
\int G(u+\phi)-G(u)>0 \text {. }
$$

Then

$$
\int \nabla u \cdot \nabla \phi+\frac{1}{2} \int|\nabla \phi|^{2} \geqq 0 .
$$

Note that $\int G(u+\phi)$ makes sense for $\phi \in \mathscr{K}$; indeed if $B=\operatorname{Supp} \phi$ then

$$
\int|G(u+\phi)| \leqq \int_{B}|G(u+\phi)|+\int_{\sim B}|G(u)| \leqq \int_{B}\left(C+C|u+\phi|^{p}\right)+\int|G(u)|<\infty .
$$

Proof. We have, with $B=\operatorname{Supp} \phi$,

$$
\int G\left(u^{j}+\phi\right)=\int_{B} G\left(u^{j}+\phi\right)+\int_{\sim B} G\left(u^{j}\right) \geqq \int_{B}\left[G\left(u^{j}+\phi\right)-G\left(u^{j}\right)\right] \rightarrow \int_{B} G(u+\phi)-G(u) .
$$

For the last assertion we note that

$$
G\left(u^{j}+\phi\right)-G\left(u^{j}\right) \rightarrow G(u+\phi)-G(u) \text { a.e. . }
$$

On the other hand, if $A \subset B$ we have

$$
\begin{aligned}
\int_{A}\left|G\left(u^{j}+\phi\right)-G\left(u^{j}\right)\right| & \leqq \int_{A}\left(C+C\left|u^{j}\right|^{p}+C|\phi|^{p}\right) \leqq C \mu(A)+C \mu(A)^{1 / 2}\left(\int_{B}\left|u^{j}\right|^{2 p}+\int_{B}|\phi|^{2 p}\right)^{1 / 2} \\
& \leqq C \mu(A)+C \mu(A)^{1 / 2}
\end{aligned}
$$

and the last term can be made arbitrarily small by choosing $\mu(A)$ small enough. We conclude the proof by Egorov's or Vitali's lemma. Thus, if (3.15) holds, we have $\int G\left(u^{j}+\phi\right)>0$ for $j$ large enough, and therefore $\frac{1}{2} \int\left|\nabla\left(u^{j}+\phi\right)\right|^{2} \geqq T$. Since $\frac{1}{2} \int\left|\nabla u^{j}\right|^{2}$ $\rightarrow T$, we obtain (3.16) in the limit.

Lemma 3.3. There is a constant $C_{1}$ (depending only on $G$ ) such that if $\phi \in \mathscr{K}$, then

$$
\left(\int g(u) \cdot \phi-C_{1} \int|\phi|[u=0]>0\right) \Longrightarrow\left(\int \nabla u \cdot \nabla \phi \geqq 0\right) .
$$

Note that $\int g(u) \phi$ makes sense since $g(u) \in L^{2}(B)$ and $\phi \in L^{2}(B)$ (here $B=\operatorname{Supp} \phi)$. On the other hand, $\int|\phi|[u=0]$ also makes sense since $\phi \in L^{1}(B)$.

Proof. By dominated convergence we have, as $t \rightarrow 0$,

$$
\begin{aligned}
\int[G(u+t \phi)-G(u)][u \neq 0] & =t \int g(u) \cdot \phi[u \neq 0]+o(t) \\
& =t \int g(u) \cdot \phi+o(t) .
\end{aligned}
$$

On the other hand we have

$$
\left|\int G(t \phi)[u=0]\right| \leqq C_{1} t \int|\phi|[u=0]+o(t)
$$

[here we use assumption (3.3) to deduce that $|G(v)| \leqq C_{1}|v|+C|v|^{p}$, all $\left.v\right]$ ). Let $\phi \in \mathscr{K}$ be such that

$$
\int g(u) \cdot \phi-C_{1} \int|\phi|[u=0]>0 .
$$

We deduce from (3.18) and (3.19) that $\int[G(u+t \phi)-G(u)]>0$ for $t>0$ and small enough. Therefore, by Lemma 3.2

$$
\int \nabla u \cdot \nabla \phi+\frac{t}{2} \int|\nabla \phi|^{2} \geqq 0
$$


As $t \rightarrow 0$ we have $\int \nabla u \cdot \nabla \phi \geqq 0$, which is precisely (3.17).

Lemma 3.4. Consider the linear functional $L(\phi)=\int \nabla u \cdot \nabla \phi$. There is some $\phi \in \mathscr{K}$ such that $L(\phi) \neq 0$ and $\phi=0$ on $[u=0]$.

Proof. Assuming the contrary, we should have $L(\phi)=0$, for all $\phi \in \mathscr{K}$ such that $\phi=0$ on $[u=0]$. In particular, taking $\phi=\left(\phi_{1}, 0, \ldots, 0\right)$, we should have $\int \nabla u_{1} \cdot \nabla \phi_{1}=0$, for all $\phi \in \mathscr{K}$ such that $\phi_{1}=0$ on $\left[u_{1}=0\right]$. We choose $\phi_{1}=\left(u_{1}-\delta\right)_{+}$, $\delta>0$. Then, from the above, $\int\left|\nabla\left(u_{1}-\delta\right)_{+}\right|^{2}=0$, which implies $\left(u_{1}-\delta\right)_{+}=C$, which in turn implies $\left(u_{1}-\delta\right)_{+} \equiv 0$ (since $u_{1} \rightarrow 0$ at infinity in the weak sense). Hence, $u_{1} \leqq \delta$, and thus $u_{1} \leqq 0$. The same argument applied to each component leads to $u$ $\equiv 0$, which is a contradiction.

Lemma 3.5. There is a constant $k \geqq 0$ such that

$$
\left|\int g(u) \phi-k L(\phi)\right| \leqq C_{1} \int|\phi|[u=0], \text { for all } \phi \in \mathscr{K} \text {. }
$$

Proof. We fix some $\phi_{0} \in \mathscr{K}$ such that $L\left(\phi_{0}\right)=-1$ and $\phi_{0}=0$ on $[u=0]$. (See Lemma 3.4.) Given $\phi \in \mathscr{K}$, note that

$$
\psi=\phi+L(\phi) \phi_{0}+\alpha \phi_{0}, \quad \alpha>0,
$$

satisfies

$$
L(\psi)=-\alpha<0
$$

and, by Lemma 3.3, we have that

$$
\int g(u) \cdot\left[\phi+L(\phi) \phi_{0}+\alpha \phi_{0}\right]-C_{1} \int|\phi|[u=0] \leqq 0
$$

(since $\phi_{0}=0$ on $[u=0]$ ). As $\alpha \rightarrow 0$ we find that

$$
\int g(u) \cdot \phi-k L(\phi)-C_{1} \int|\phi|[u=0] \leqq 0, \text { for all } \phi \in \mathscr{K},
$$

where $k=-\int g(u) \cdot \phi_{0} \geqq 0$ [by (3.17)]. By considering the two choices $\pm \phi$, we obtain (3.21).

Lemma 3.6. For $v \in H_{\text {loc }}^{1}$ we have that

$$
\partial G(v) / \partial x_{i}=g(v) \cdot \partial v / \partial x_{i} \text { in } \mathscr{D}^{\prime} .
$$

Note that $g(v) \cdot \partial v / \partial x_{i} \in L_{\text {loc }}^{1}$ and $G(v) \in L^{1}$, so that (3.22) makes sense in $\mathscr{D}^{\prime}$. Proof. Choose a smoothing sequence $G_{k}$ for $G$ so that $G_{k} \rightarrow G$ pointwise on $\mathbb{R}^{n}$, $g_{k}=\nabla G_{k}$ tends to $g$ pointwise on $\mathbb{R}^{n} \backslash\{0\}$. Moreover,

$$
\left|G_{k}(v)\right| \leqq C|v|+C|v|^{p} \quad \text { and } \quad\left|g_{k}(v)\right| \leqq C+C|v|^{p-1} .
$$

We have that $\partial G_{k}(v) / \partial x_{i}=g_{k}(v) \cdot \partial v / \partial x_{i}$, and thus, for $\phi \in C_{0}^{\infty}$,

$$
\int \phi \partial G_{k}(v) / \partial x_{i}=-\int G_{k}(v) \partial \phi / \partial x_{i} \rightarrow-\int G(v) \partial \phi / \partial x_{i},
$$

and

$$
\int \phi g_{k}(v) \cdot \partial v / \partial x_{i} \rightarrow \int \phi g(v) \cdot \partial v / \partial x_{i},
$$

by dominated convergence (recall that $\partial v / \partial x_{i}=0$ a.e. on the set $[v=0]$ ). 
Proof of Theorem 1 Concluded. The linear functional $M(\phi)=\int g(u) \cdot \phi-k L(\phi)$, $\phi \in C_{0}^{\infty}$, satisfies, by (3.21), $|M(\phi)| \leqq C_{1}\|\phi\|_{L^{1}}$. Thus by the Riesz representation theorem there is some function $h \in L^{\infty}\left(\mathbb{R}^{2}\right), h: \mathbb{R}^{2} \rightarrow \mathbb{R}^{n}$, such that $M(\phi)=\int h \cdot \phi$, for all $\phi \in C_{0}^{\infty}$. Moreover, by (3.21), we have $\left|\int h \cdot \phi\right| \leqq C \int|\phi|[u=0]$, for all $\phi \in C_{0}^{\infty}$, and hence for all $\phi \in L^{1}$. Thus, $h=0$ for $u \neq 0$ and, therefore, $g(u)+k \Delta u=[u=0] h$. It follows that $k \neq 0$ (and thus $k>0$ ), for otherwise $k=0 \Rightarrow g(u) \equiv 0 \Rightarrow \partial G(u) / \partial x_{i}=0$ by Lemma $3.6 \Rightarrow G(u)=C \Rightarrow G(u) \equiv 0\left(\right.$ since $\left.G(u) \in L^{1}\right) \Rightarrow$ for a.e. $x$ we have either $u(x)=0$ or $|u(x)| \geqq \varepsilon$. On the other hand, $|u| \in H_{\text {loc }}^{1}$, and thus it has a mean value property; therefore we would have either $u \equiv 0$ a.e. on $\mathbb{R}^{2}$ or $|u| \geqq \varepsilon$ a.e. on $\mathbb{R}^{2}$. Both cases are excluded (since $u \neq 0$ ). Hence we have proved that $k>0$ and $u$ satisfies $-\Delta u=\frac{1}{k} g(u)+[u=0] h^{\prime}$ for some $h^{\prime} \in L^{\infty}$. It follows from the elliptic regularity theory that $u \in W_{\text {loc }}^{2, q}$, all $q<\infty$, and therefore $\Delta u=0$ a.e. on $[u=0]$. Consequently $h^{\prime}=0$ a.e. on $[u=0]$, i.e. we have

$$
-\Delta u=g(u) / k \text { for some } k>0 \text {. }
$$

When $d=2$, Pohozaev's identity (the proof of which is similar to Lemma 2.4) states that $\int G(u)=0$. On the other hand, since $\nabla u^{j} \rightarrow \nabla u$ weakly in $L^{2}$, we have, by lower semicontinuity, $\frac{1}{2} \int|\nabla u|^{2} \leqq T$. Thus, in fact, $\frac{1}{2} \int|\nabla u|^{2}=T$ and $u$ is a minimizer for (3.4). After scaling we can always assume that $u$ also satisfies $-\Delta u=g(u)$. Finally, if $v \in \mathscr{C}$ satisfies $-\Delta v=g(v)$ in $\mathscr{D}^{\prime}$, then $v \in L_{\mathrm{loc}}^{q}$, all $q<\infty$, $\Rightarrow g(v) \in L_{\mathrm{loc}}^{q} \Rightarrow v \in L_{\mathrm{loc}}^{\infty}$. By Pohozaev's identity we have $\int G(v)=0$, and thus if $v \neq 0$ we obtain $\frac{1}{2} \int|\nabla v|^{2} \geqq T$. Therefore,

$$
S(v)=\frac{1}{2} \int|\nabla v|^{2} \geqq T=\frac{1}{2} \int|\nabla u|^{2}=S(u) .
$$

Behavior at Infinity

Here we assume only that $g: \mathbb{R}^{n} \rightarrow \mathbb{R}^{n}$ is any mapping such that for some $p<\infty$,

$$
|g(v)| \leqq C+C|v|^{p-1}, \text { for all } v \in \mathbb{R}^{n} .
$$

Theorem 3.2. Let $v \in \mathscr{C}$ be any solution of $(1.1)$ in $\mathscr{D}^{\prime}$. Then

$$
\lim _{|x| \rightarrow \infty} v(x)=0 .
$$

Assume in addition that $g(v) \cdot v \leqq-C|v|^{r}$ for all $v \in \mathbb{R}^{n}$ with $|v|<\delta$ for some constants $C>0, \delta>0,1 \leqq r \leqq 2$. Then

(i) if $r=2, v(x)$ decays exponentially as $|x| \rightarrow \infty$,

(ii) if $1 \leqq r<2, v(x)$ has compact support.

Proof. For any $\delta>0$ the function $\phi=(|v|-\delta)_{+}$satisfies $\phi \in L_{\mathrm{loc}}^{1}, \nabla \phi \in L^{2}$, $\mu(\operatorname{Supp} \phi)<\infty$, and thus, by $(3.10), \phi \in L^{q}\left(\mathbb{R}^{2}\right)$ for all $q<\infty$. Hence $\int|v|^{q}[|v|>\delta]<\infty$, for all $q<\infty$, all $\delta>0$. We note that

$$
-\Delta|v|^{2}=-2 v \cdot \Delta v-2|\nabla v|^{2} \leqq 2 v \cdot g(v) \leqq C|v|+C|v|^{p}
$$

Given any $\alpha>0$, we have, for some $\delta>0$,

$$
-\Delta|v|^{2}+|v|^{2} \leqq C|v|+|v|^{2}+C|v|^{p} \leqq \alpha+C\left(|v|^{2}+|v|^{p}\right)[|v|>\delta],
$$


and thus $|v|^{2} \leqq C \alpha+(Y * \psi)$, where $Y$ is the Yukawa (or Bessel) potential and $\psi=C\left(|v|^{2}+|v|^{p}\right)[|v|>\delta]$. Thus $\psi \in L^{q}$, all $1 \leqq q<\infty$. On the other hand $Y \in L^{t}$ for all $1 \leqq t<\infty$. It follows that $(Y * \psi) \rightarrow 0$ as $|x| \rightarrow \infty$. Therefore,

$$
\limsup _{|x| \rightarrow \infty}|v(x)|^{2} \leqq C,
$$

which implies that $v(x) \rightarrow 0$ as $|x| \rightarrow \infty$, since $\alpha$ is arbitrary.

The rest of the proof is the same as in Theorem 2.3.

\section{Appendix}

Lemma A.1. Let $1<\alpha<\beta<\infty$ and $1 / \alpha+1 / \alpha^{\prime}=1$. Let $Y \in L_{w}^{\alpha}\left(\right.$ weak $\left.L^{\alpha}\right), A \in L^{\alpha^{\prime}}$ with $\mu(\operatorname{Supp} A)<\infty$, and $f \in L^{\beta}$ with $A, Y, f \geqq 0$. We assume that $f \leqq 1+Y *(A f)$, where * denotes convolution. Then

$$
\int_{B}|f|^{q}<\infty
$$

for all $q<\infty$ and all $B$ of finite measure.

Proof. Let $\chi$ be the characteristic function of $B \cup \operatorname{Supp} A$. We have $\chi f \leqq \chi+\chi[Y * A \chi f]$. Let $g=\chi f$, whence $g \leqq \chi+\chi[Y *(A g)]$, and $g \in L^{\beta}$ with $\mu(\operatorname{Supp} g)<\infty$. Let $Q: \phi \mapsto Y *(A \phi)$. Note that $Q$ is a well defined bounded operator from $L^{\gamma}$ into $L^{\gamma}$ for all $\alpha<\gamma<\infty$, with norm $\leqq C_{\gamma}\|Y\|_{\alpha, w}\|A\|_{\alpha^{\prime}}$. We introduce $\beta_{1}$ defined by

$$
1 / \beta_{1}= \begin{cases}1 / \beta-1 / \alpha^{\prime}, & \text { if } \beta<\alpha^{\prime}, \\ 1 /(\beta+1), & \text { if } \beta \geqq \alpha^{\prime} .\end{cases}
$$

Note that $\beta_{1}>\beta$. We shall prove that $g \in L^{\beta} \Rightarrow g \in L^{\beta_{1}}$. Iterating this fact with $\beta$ replaced by $\beta_{1}$ we find that $g \in L^{\beta_{k}}$ for an increasing sequence $\beta_{k} \rightarrow \infty$. This will prove the lemma. Write $A=A_{1}+A_{2}$ with $A_{1} \in L^{\infty}$ and $A_{2}$ such that $K: \phi \mapsto Y *\left(A_{2} \phi\right)$ is a bounded operator from $L^{\beta}$ into $L^{\beta}$ and $L^{\beta_{1}}$ into $L^{\beta_{1}}$ with norm $<1$. We have that

$$
g \leqq\left[\chi+\chi\left(Y *\left(A_{1} g\right)\right)\right]+\left[Y *\left(A_{2} g\right)\right] \equiv h+K g .
$$

Note that $h \in L^{\beta_{1}}$. We have that

$$
g \leqq \sum_{j=1}^{m} K^{j} h+K^{m+1} g .
$$

$K^{j} h$ is a norm convergent series in $L^{\beta_{1}}$ while $K^{m+1} g \rightarrow 0$ in $L^{\beta}$. Thus $g \in L^{\beta_{1}}$.

Lemma A.1 is closely related to, and in fact implies some results in [8].

\section{References}

1. Affleck, I.: Two dimensional disorder in the presence of a uniform magnetic field. J.Phys. C 16, 5839-5848 (1983)

2. Benilan, Ph., Brezis, H., Crandall, M.: A semilinear equation in $L^{1}\left(\mathbb{R}^{N}\right)$. Ann. Scuola Norm. Sup. Pisa 2, 523-555 (1975) 
3. Berestycki, H., Gallouet, Th., Kavian, O.: Equations de champs scalaires Euclidiens non linéaires dans le plan. Compt. Rend. Acad. Sci. 297, 307-310 (1983)

4. Berestycki, H., Gallouet, Th., Kavian, O.: Semilinear elliptic problems in $\mathbb{R}^{2}$ (in preparation)

5. Berestycki, H., Lions, P.-L.: Existence of stationary states of non-linear scalar field equations. In: Bifurcation phenomena in mathematical physics and related topics. Bardos, C., Bessis, D. (eds.). Proc. NATO ASI, Cargese, 1979, Reidel, 1980

6. Berestycki, H., Lions, P.-L.: Nonlinear scalar field equations. I. Existence of a ground state. 84, 313-345 (1983). See also II: Existence of infinitely many solutions. Arch. Rat. Mech. Anal. 84, 347-375 (1983). See also An O.D.E. approach to the existence of positive solutions for semilinear problems in $\mathbb{R}^{N}$ (with L.A. Peletier). Ind. Univ. Math. J. 30, 141-157 (1981). See also Une méthode locale pour l'existence de solutions positives de problèmes semilinéaires elliptiques dans $\mathbb{R}^{N}$. J. Anal. Math. 38, 144-187 (1980)

7. Berestycki, H., Lions, P.-L.: Existence d'états multiples dans les équations de champs scalaires non linéaires dans le cas de masse nulle. Compt. Rend. Acad. Sci. 297, I, 267-270 (1983)

8. Brezis, H., Kato, T.: Remarks on the Schrödinger operator with singular complex potentials. J. Math. Pures Appl. 58, 137-151 (1979)

9. Brezis, H., Lieb, E.H.: A relation between pointwise convergence of functions and convergence of functionals. Proc. Am. Math. Soc. 88, 486-490 (1983)

10. Coleman, S., Glaser, V., Martin, A.: Action minima among solutions to a class of Euclidean scalar field equation. Commun. Math. Phys. 58, 211-221 (1978)

11. Gilbarg, D., Trudinger, N.S.: Elliptic partial differential equations of second order. Berlin, Heidelberg, New York: Springer 1977

12. Kato, T.: Schrödinger operators with singular potentials. Israel J. Math. 13, 135-148 (1972)

13. Lieb, E.H.: Some vector field equations. In: Differential equations. Proc. of the Conference Held at the University of Alabama in Birmingham, USA, March 1983, Knowles, I., Lewis, R. (eds.). Math. Studies Series, Vol. 92. Amsterdam: North-Holland 1984

14. Lieb, E.H.: On the lowest eigenvalue of the Laplacian for the intersection of two domains. Invent. Math. 74, 441-448 (1983)

15. Lions, P.-L.: Principe de concentration-compacité en calcul des variations. Compt. Rend. Acad. Sci. 294, 261-264 (1982)

16. Lions, P.-L.: The concentration-compactness principle in the calculus of variations: The locally compact case, Parts I and II. Ann. Inst. H. Poincaré, Anal. Non-lin. (submitted)

17. Nirenberg, L.: On elliptic partial differential equations. Ann. Scuola Norm. Sup. Pisa 13, $115-162$ (1959)

18. Pohozaev, S.I.: Eigenfunctions of the equation $\Delta u+\lambda f(u)=0$. Sov. Math. Dokl. 6, 1408-1411 (1965)

19. Stampacchia, G.: Equations élliptiques du second ordre à coéfficients discontinus. Montréal: Presses de l'Université de Montréal 1966

20. Strauss, W.A.: Existence of solitary waves in higher dimensions. Commun. Math. Phys. 55, 149-162 (1977)

21. Keller, C.: Large-time asymptotic behavior of solutions of nonlinear wave equations perturbed from a stationary ground state. Commun. Partial Diff. Equations 8, 1013-1099 (1983)

22. Strauss, W.A., Vázquez, L.: Existence of localized solutions for certain model field theories. J. Math. Phys. 22, 1005-1009 (1981)

23. Diaz, J.I.: Nonlinear partial differential equations and free boundaries. London: Pitman (in preparation)

Communicated by A. Jaffe

Received March 30, 1984; in revised form May 18, 1984 
